\title{
Diagnósticos de enfermagem em portadores da Síndrome da Imunodeficiência AdQuirida
}

\author{
Nursing diagnoses for patients with AcQuired Immunodeficiency Syndrome \\ Diagnósticos de enfermería em portadores del Sindrome de Inmunodeficiencia AdQuirida
}

\author{
Myria Ribeiro da Silva', Ana Rita de Cássia Bettencourt', \\ Solange Diccini', Angélica Belasco', Dulce Aparecida Barbosa' \\ 'Universidade Federal de São Paulo. Departamento de Enfermagem. São Paulo, SP
}

Submissão: 12/04/2008

Aprovação: 09/12/2008

\begin{abstract}
RESUMO
Os objetivos deste estudo foram identificar os diagnósticos de enfermagem mais freeüentes segundo a taxonomia II da NANDA em pacientes adultos portadores de HIV/aids e verificar com Que freqüência as características definidoras, os fatores relacionados e de risco deram suporte para a elaboração destes. O levantamento dos dados foi realizado pelos pesquisadores nos 60 pacientes internados com HIV/aids e depois feita a verificação se os requisitos propostos pela NANDA para realização dos DE eram adequados. Foram selecionados 13 dos 38 diagnósticos com freeüência igual ou superior a $80 \%$. Os resultados indicaram concordância e adeQuações para identificação. Porém, novos estudos serão necessários tanto para confirmação dos achados, assim como trazer novos conhecimentos ao assunto e estimular a pesQuisa nesta população de pacientes.
\end{abstract}

Descritores: Enfermagem; Diagnóstico de enfermagem; AIDS.

\begin{abstract}
This study aims at identifying the most frequent nursing diagnoses, according to the NANDA's taxonomy II in adult patients of HIV/ AIDS and to check how often the defining characteristics, the related factors and the risk factors provided support for their development. Data survey was conducted by researchers in the 60 patients admitted with HIV/aids, followed by the finding out whether the NANDA's proposed requirements to develop the Nursing Diagnoses were appropriate. From the 38 diagnoses, 13 diagnoses were chosen with a frequency equal or higher than $80 \%$. Results pointed to agreement and appropriateness for identification. But new studies will be necessary either to acknowledge the findings and bring new knowledge to the topic as well, besides encouraging research with this patients' population.
\end{abstract}

Descriptors: Nursing; Nursing diagnosis; AIDS.

\section{RESUMEN}

Los objetivos del estudio fuerón identificar los diagnósticos de enfermería según la taxonomia de Nanda en pacientes adultos portadores de HIV/Sida y observar con Que frecuencia las caracteristicas que son definidas por los factores relacionados pelos investigadores en los 60 pacientes internados con VIH/SIDA y despúes hecha la verificación de los reQuisitos propuestos en Nanda para realización de los diagnósticos de la enfermeria que eran adequados. Fuerón relacionados 13 de los 38 diagnósticos con frecuencia igual al superior de $80 \%$. Los resultados indican concordancia y sentido para identificar. Pues nuevos estudios fuerón necesarios tanto para a confirmación de los hallazgos asin como traer nuevos conhecimentos sobre el asunto y estimular a investigación neste grupo de pacientes.

Descriptores: Enfermería; Diagnóstico de enfermería; SIDA. 


\section{INTRODUÇÃO}

Em todo o mundo a disseminação do vírus da imunodeficiência humana (HIV) continua em ritmo alarmante. Essa pandemia criou um impacto dramático e freqüentemente devastador em muitos países. Embora muito se tenha aprendido sobre essa doença, os pesquisadores não têm previsão de cura no futuro imediato, prevendo-se Que seja crescente o número de indivíduos infectados pelo HIV ${ }^{(1)}$.

No Brasil, no início dos anos 80 , a epidemia afetava principalmente homo/bissexual masculinos, brancos e de classe média ou alta, habitantes das grandes metrópoles. Na segunda década da epidemia, homens heterossexuais, mulheres, crianças e todas as classes sociais estão sendo atingidos. Os novos dados confirmam a tendência de Queda na epidemia no Brasil, mas desperta para a urgência de ações estratégicas em determinadas regiões do país e populações vulneráveis ${ }^{(2)}$.

Durante muitos anos, a enfermagem teve uma orientação dirigida para enfrentar as situações imediatas de modo espontâneo, intuitivo e tradicional. Sob a influência de vários fatores, o agir da enfermeira foi se constituindo de procedimentos descritos como a arte de enfermagem, a Qual, nas primeiras décadas do século $X X$, se organiza de modo sistematizado em técnicas de enfermagem ${ }^{(3)}$.

A responsabilidade do cuidar em enfermagem exige Que as decisões sobre as intervenções propostas sejam fundamentadas na avaliação do estado de saúde do indivíduo. Essa avaliação reQuer Que se adote o diagnóstico de enfermagem como referência ${ }^{(4)}$.

Uma terminologia própria facilita a comunicação oral e escrita entre os enfermeiros, proporcionando uma linguagem comum a todos. A identificação dos diagnósticos proporciona intervenções adequadas, estimulando o desenvolvimento do conhecimento e de habilidades para o atendimento das necessidades do ser humano ${ }^{(5)}$.

Identificar diagnósticos de enfermagem envolve a ativação de uma série de habilidades mentais Que permitem Que façamos inferências sobre dados observáveis. Dados observáveis podem ser entendidos como peças de informação Que obtemos por entrevista, exame físico e outros. Com isso, o diagnóstico, além de ser definido como uma das etapas do processo de enfermagem pode ser definido como um processo de julgamento clínico, o Qual é tradicional na prática da mesma ${ }^{(6)}$.

A Taxonomia II da NANDA ${ }^{(7)}$ apresenta termos cujos significados são mais familiares, fazem parte da enfermagem tradicional e contemporânea e talvez, sejam mais efetivos para a comunicação com os outros profissionais, com o próprio cliente e com instâncias de planejamento dos serviços de saúde ${ }^{(4)}$.

Ao utilizar o diagnóstico de enfermagem como uma parte do processo de enfermagem, a enfermagem profissional identifica um corpo de conhecimentos Que contribui para a prevenção da doença, bem como para a manutenção e/ou restabelecimento da saúde (ou alívio da dor e do desconforto, Quando um retorno à saúde não é mais possível). Uma vez Que o processo de enfermagem constitui a base de todas as ações de enfermagem, trata-se da essência da enfermagem. O processo é flexível, ainda Que suficientemente estruturado de modo a proporcionar o embasamento para as ações de enfermagem ${ }^{(8)}$.

O uso do diagnóstico de enfermagem tanto no ensino como na prática e na pesquisa é uma necessidade Que cada vez mais se torna emergente para a profissão, representa uma forma de raciocínio lógico Que possibilita uma inter-relação de causas e efeitos das alterações apresentadas pelo paciente. Facilita o estabelecimento de metas, a adoção de condutas de enfermagem e a realização da avaliação da assistência prestada.

Apesar dos progressos feitos no tratamento da infecção por HIV e da aids, o vírus persiste como uma Questão crítica de saúde. Prevenção, detecção precoce e tratamento permanecem como importantes aspectos do cuidado de pessoas com infecção por este vírus. As enfermeiras em todos os setores encontram pessoas com essa doença; assim, as enfermeiras precisam da compreensão sobre o distúrbio, de conhecimento sobre as conseqüências físicas e psicológicas associadas a seu diagnóstico e de habilidades experientes Quanto ao histórico e tratamento clínico para proporcionar um cuidado para as pessoas com infecção por HIV e AIDS.

Tendo em vista a complexidade da doença e a morbidade associada, ficamos motivados a realização deste estudo Que tem como objetivo identificar os Diagnósticos de Enfermagem mais freeüentes nos pacientes portadores de HIV/aids e os fatores de risco e fatores relacionados Que deram suporte para elaboração dos mesmos.

\section{MÉTODO}

\section{Local, Período do Estudo e Aspectos Éticos}

O estudo foi realizado no Hospital São Paulo (HSP) / Universidade Federal de São Paulo (UNIFESP), na unidade de internação da disciplina de Doenças Infecciosas e Parasitárias do Adulto, no ano de 2006, após aprovação do Comitê de Ética em Pesquisa da Universidade Federal de São Paulo/UNIFESP e assinatura do termo de consentimento livre e esclarecido dos incluídos no estudo.

\section{População e Amostra}

A população $(n=60)$ constituiu-se de todos os pacientes portadores de HIV/aids, hospitalizados na unidade de internação da Disciplina de doenças infecciosas e parasitárias/adulto Que concordaram em participar do estudo.

\section{Procedimento de Coleta de Dados}

Para todo paciente internado foi realizado a coleta dos dados Que se constituiu dos dados sócio demográficos, histórico, exame físico e dos diagnósticos de enfermagem que foram identificados através das características definidoras e fatores de risco e relacionado. A seguir foi feita uma análise para verificar com Que freqüência as características definidoras, fatores de risco e relacionados propostos pela NANDA estavam adequados para realização dos DE nesta população de pacientes. O instrumento de coleta de dados utilizado foi um modelo de registro de enfermagem implantado no Hospital São Paulo, Que utiliza os referenciais de Horta, Orem, epidemiológicos de risco e biomédicos, Que contém dados da entrevista, do exame físico e dados de exames laboratoriais.

\section{RESULTADOS}

Neste estudo, as micobacterioses (55\%) foram as infecções oportunistas de maior prevalência, seguida das neuroinfecções $(38,3 \%)$. 
Tabela 1. Características sócio-demográficas dos pacientes HIV positivos internados na unidade DIPA.

\begin{tabular}{|c|c|c|c|}
\hline Características & $\begin{array}{c}\text { Masculino } \\
n(\%)\end{array}$ & $\begin{array}{c}\text { Feminino } \\
n(\%)\end{array}$ & $\begin{array}{l}\text { Total } \\
\text { n (\%) }\end{array}$ \\
\hline Sexo & 39 & 21 & 60 \\
\hline Média de idade (anos) & 36 & 35 & 35,5 \\
\hline \multicolumn{4}{|l|}{ Escolaridade } \\
\hline $2^{\circ}$ grau completo & $16(41,0 \%)$ & $07(33,3 \%)$ & $23(38,3 \%)$ \\
\hline \multicolumn{4}{|l|}{ Estado civil } \\
\hline Solteiro & $25(64,1 \%)$ & $09(42,8 \%)$ & $34(56,6 \%)$ \\
\hline Casado & $09(23,0 \%)$ & $08(38,1 \%)$ & $17(28,3 \%)$ \\
\hline \multicolumn{4}{|l|}{ Drogas } \\
\hline Não injetáveis* & $11(28,2 \%)$ & $03(14,3 \%)$ & $14(23,3 \%)$ \\
\hline Injetáveis** & $08(20,5 \%)$ & $03(14,3 \%)$ & $11(18,3 \%)$ \\
\hline Nenhuma & $20(51,3 \%)$ & $15(71,4 \%)$ & $35(58,3 \%)$ \\
\hline
\end{tabular}

Tabela 2. Características definidoras, fatores de risco e fatores relacionados dos Diagnósticos de Enfermagem do domínio Segurança e Proteção, identificados nos pacientes HIV positivos internados na unidade DIPA.

\begin{tabular}{|c|c|c|}
\hline Diagnósticos de Enfermagem / Fatores de risco e relacionados & $\mathrm{n}$ & $\%$ \\
\hline Risco de Infecção & 60 & 100,0 \\
\hline \multicolumn{3}{|l|}{ Fatores de risco } \\
\hline Procedimentos invasivos & 60 & 100,0 \\
\hline Defesas secundárias inadeQuadas & 60 & 100,0 \\
\hline Imunossupressão & 60 & 100,0 \\
\hline Proteção Ineficaz & 60 & 100,0 \\
\hline \multicolumn{3}{|l|}{ Características Definidoras } \\
\hline Imunidade deficiente & 60 & 100,0 \\
\hline Dispnéia & 32 & 53,3 \\
\hline Desorientação & 27 & 45,0 \\
\hline \multicolumn{3}{|l|}{ Fatores relacionados } \\
\hline Perfis sangüíneos alterados & 60 & 100,0 \\
\hline Distúrbios imunológicos & 60 & 100,0 \\
\hline Nutrição inadeQuada & 36 & 60,0 \\
\hline$\underline{\text { Risco de Integridade da Pele Prejudicada }}$ & 52 & 86,6 \\
\hline \multicolumn{3}{|l|}{ Fatores de risco } \\
\hline \multicolumn{3}{|l|}{ Externos: } \\
\hline Hipertermia & 26 & 50,0 \\
\hline Imobilização física, fatores mecânicos (contenção) & 20 & 38,5 \\
\hline \multicolumn{3}{|l|}{ Internos: } \\
\hline Fatores imunológicos & 52 & 100,0 \\
\hline Alteração no estado nutricional (emagrecimento) & 36 & 69,2 \\
\hline Alterações no turgor da pele & 28 & 53,8 \\
\hline Medicação & 19 & 36,5 \\
\hline Proeminência esquelética & 15 & 28,8 \\
\hline Risco de Lesão & 49 & 81,6 \\
\hline \multicolumn{3}{|l|}{ Fatores de risco } \\
\hline \multicolumn{3}{|l|}{ Externos: } \\
\hline Modo de transportar ou de transporte & 19 & 38,8 \\
\hline \multicolumn{3}{|l|}{ Internos: } \\
\hline Disfunção auto-imune & 49 & 100,0 \\
\hline Má nutrição & 36 & 73,5 \\
\hline Físicos (pele lesada, mobilidade alterada) & 16 & 32,6 \\
\hline
\end{tabular}

Foram identificados 38 diagnósticos de enfermagem, sendo Que optamos por apresentar os 13 diagnosticados Que apareceram com freqüência acima de $80 \%$ nos pacientes estudados.
A seguir, serão apresentadas as características definidoras, os fatores de risco e fatores relacionados de acordo com os padrões funcionais de saúde propostos pela NANDA, Que deram suporte 
para elaboração destes diagnósticos de enfermagem com freQüência superior a $80 \%$.

O diagnóstico risco para infecção acometeu 100\% dos pacientes e foi evidenciado pelos seguintes fatores de risco: procedimentos invasivos, defesas secundárias inadeQuadas e imunossupressão (100\%).

Quanto à proteção ineficaz presente em $100 \%$ dos casos, as características definidoras presentes tem como a imunidade deficiente em 100\%, dispnéia em 53,3\%, desorientação em 45\% e frąueza e agitação em $31,6 \%$ e os fatores relacionados foram: perfis sanguíneos alterados e distúrbios imunológicos (100\%) e nutrição inadeQuada em $60 \%$.
Em relação ao diagnóstico risco de integridade da pele prejudicada evidenciado em 52 pacientes $(86,6 \%)$ os fatores de risco internos encontrados foram: fatores imunológicos em 100\% dos pacientes estudados e alteração no estado nutricional em 69,2\% e como fator de risco externo a hipertermia, presente em 26 (50\%) dos casos.

Os fatores de risco evidenciados para o diagnóstico risco de lesão Que esteve presente em $49(81,6 \%)$ dos pacientes foram identificados como principais fatores internos a disfunção autoimune em $100 \%$ e má nutrição em 73,5\% dos pacientes.

O diagnóstico padrão de sono perturbado foi identificado em

Tabela 3. Características definidoras, fatores de risco e fatores relacionados dos Diagnósticos de Enfermagem do domínio Atividade/Repouso, identificados nos pacientes HIV positivos internados na unidade DIPA.

\begin{tabular}{|c|c|c|}
\hline Diagnósticos de Enfermagem / Fatores de risco e relacionados & $\mathrm{n}$ & $\%$ \\
\hline Padrão de Sono Perturbado & 60 & 100,0 \\
\hline \multicolumn{3}{|l|}{ Características definidoras } \\
\hline Três ou mais despertares à noite & 29 & 48,3 \\
\hline Preocupação em tentar dormir & 26 & 43,3 \\
\hline Insatisfação com o sono & 25 & 41,6 \\
\hline \multicolumn{3}{|l|}{ Fatores relacionados } \\
\hline \multicolumn{3}{|l|}{ Psicológicos } \\
\hline Medicações & 35 & 58,3 \\
\hline Preocupação de tentar dormir & 26 & 43,3 \\
\hline Separação de pessoas significativas & 17 & 28,3 \\
\hline \multicolumn{3}{|l|}{ Ambientais } \\
\hline Despertar causado por outros & 35 & 58,3 \\
\hline Enfermeira com medicação, controle ou exames laboratoriais & 35 & 58,3 \\
\hline \multicolumn{3}{|l|}{ Fisiológicos } \\
\hline Febre & 26 & 43,3 \\
\hline Náusea & 22 & 36,6 \\
\hline Falta de ar & 14 & 23,3 \\
\hline Intolerância à Atividade & 56 & 93,3 \\
\hline \multicolumn{3}{|l|}{ Características definidoras } \\
\hline Desconforto ou dispnéia de esforço & 42 & 70 \\
\hline Resposta anormal da pressão sanguínea & 20 & 33,3 \\
\hline \multicolumn{3}{|l|}{ Fatores relacionados } \\
\hline Desequilíbrio oferta e demanda de $\mathrm{O} 2$ & 41 & 73,2 \\
\hline Fraeueza generalizada & 24 & 42,8 \\
\hline Repouso no leito ou imobilidade & 20 & 35,7 \\
\hline Déficit no autocuidado para alimentação & 55 & 91,6 \\
\hline \multicolumn{3}{|l|}{ Características definidoras } \\
\hline Manusear utensílios, manipular alimentos na boca & 26 & 43,3 \\
\hline Preparar alimentos para ingestão & 25 & 41,6 \\
\hline \multicolumn{3}{|l|}{ Fatores relacionados } \\
\hline Prejuízo perceptivo ou cognitivo & 28 & 46,6 \\
\hline Prejuízo neuromuscular & 15 & 25,0 \\
\hline Dor & 15 & 25,0 \\
\hline Prejuízos musculoesqueléticos & 14 & 23,3 \\
\hline$\underline{\text { Padrão Respiratório Ineficaz }}$ & 51 & 85,0 \\
\hline \multicolumn{3}{|l|}{ Características definidoras } \\
\hline Pressão inspiratória e expiratória diminuída & 42 & 70,0 \\
\hline Dispnéia & 31 & 51,6 \\
\hline \multicolumn{3}{|l|}{ Fatores relacionados } \\
\hline Síndrome da hipoventilação & 31 & 51,6 \\
\hline Dano de percepção/cognição & 27 & 45,0 \\
\hline Disfunção neuromuscular & 24 & 40,0 \\
\hline
\end{tabular}


60 (100\%) dos pacientes estudados e tem como características definidoras principais, três ou mais despertares à noite em 48,3\% e preocupação em tentar dormir em 43,3\% e os fatores relacionados mais encontrados foram: fatores psicológicos, tais como, medicações em 58,3\% e preocupação de tentar dormir em 43,3\%; no aspecto ambiental foram relacionados: o despertar causado por outros e a enfermeira portando medicamentos, controle ou exames laboratoriais em $58,3 \%$ e no aspecto fisiológico o principal fator relacionado foi febre em $43,3 \%$.

Em relação ao diagnóstico, intolerância à atividade, em 56 $(93,3 \%)$, descreve como principal característica definidora: desconforto ou dispnéia de esforço em $70 \%$ e o fator relacionado fortemente encontrado foi o desequilíbrio entre oferta e demanda de $\mathrm{O}_{2}$, em $73,2 \%$.

Quanto ao déficit no autocuidado para alimentação Que apresentou uma freqüência de 55 (91,6\%), e apresenta como característica definidora evidente manusear utensílios, manipular alimentos na boca e segurar xícaras ou copos em 43,3\% e o fator relacionado prejuízo perceptivo ou cognitivo esteve presente em $50,9 \%$.

Para o Diagnóstico padrão respiratório ineficaz em 51 pacientes (85\%), cita como características definidoras a pressão inspiratória e expiratória diminuída em 70\% e dispnéia em 51,6\%, o principal fator relacionado foi a síndrome da hipoventilação presente em $51,6 \%$.

Para o diagnóstico conhecimento deficiente presente em 53 $(88,3 \%)$, apresenta como características definidoras principais, os comportamentos impróprios exagerados em 45\% (apatia, hostilidade) e os fatores relacionados evidenciados neste estudo foram: falta de familiaridade com recursos de informação em 56,6\% e limitação cognitiva em 50,9\%.

Tabela 4. Características definidoras, fatores de risco e fatores relacionados dos Diagnósticos de Enfermagem do domínio Percepção/Cognição e Enfrentamento/tolerância ao estresse identificados nos pacientes HIV positivos internados na unidade DIPA.

\begin{tabular}{|c|c|c|}
\hline Diagnósticos de Enfermagem / Fatores de risco e relacionados & $\mathrm{n}$ & $\%$ \\
\hline Conhecimento Deficiente & 53 & 88,3 \\
\hline \multicolumn{3}{|l|}{ Características definidoras } \\
\hline Comportamentos impróprios exagerados & 27 & 45,0 \\
\hline \multicolumn{3}{|l|}{ Fatores relacionados } \\
\hline Falta de familiaridade com os recursos de informação & 30 & 56,6 \\
\hline Limitação cognitiva & 27 & 50,9 \\
\hline Comunicação Verbal Prejudicada & 48 & 80,0 \\
\hline \multicolumn{3}{|l|}{ Características definidoras } \\
\hline Dispnéia & 30 & 50,0 \\
\hline Desorientação nas esferas de tempo espaço/pessoa & 24 & 40,0 \\
\hline Fala ou verbaliza com dificuldade & 28 & 46,6 \\
\hline \multicolumn{3}{|l|}{ Fatores relacionados } \\
\hline Alterações do SNC & 25 & 52,0 \\
\hline Percepções alteradas & 24 & 50,0 \\
\hline Confusão Aguda & 48 & 80,0 \\
\hline \multicolumn{3}{|l|}{ Características definidoras } \\
\hline Percepções errôneas & 29 & 48,3 \\
\hline Flutuação na cognição & 26 & 43,3 \\
\hline \multicolumn{3}{|l|}{ Fatores relacionados } \\
\hline Delírio & 22 & 45,8 \\
\hline Abuso de drogas & 18 & 37,5 \\
\hline Demência & 8 & 16,6 \\
\hline Ansiedade & 54 & 90,0 \\
\hline \multicolumn{3}{|l|}{ Características definidoras } \\
\hline Ansioso & 25 & 41,6 \\
\hline Nervosismo e irritabilidade & 19 & 31,6 \\
\hline \multicolumn{3}{|l|}{ Fatores relacionados: } \\
\hline Ameaça de morte & 36 & 66,6 \\
\hline Ameaça/mudança no estado de saúde & 18 & 33,3 \\
\hline Medo & 50 & 83,3 \\
\hline \multicolumn{3}{|l|}{ Características definidoras } \\
\hline Tensão aumentada & 26 & 43,3 \\
\hline Nervosismo & 23 & 38,3 \\
\hline \multicolumn{3}{|l|}{ Fatores relacionados } \\
\hline Separação do sistema de apoio em situação estressante & 37 & 74,0 \\
\hline Falta de familiaridade com experiência ambiental & 18 & 36,0 \\
\hline
\end{tabular}


Para o diagnóstico de enfermagem ansiedade, presente em 54 (90\%), cita como características definidoras na área afetiva a ansiedade em 41,6\%; e foi evidenciado pelos seguintes fatores relacionados: ameaça de morte em $66,6 \%$ e mudança e/ou ameaça no estado de saúde em 33,3\%.

Em relação ao diagnóstico de enfermagem medo em 50 pacientes $(83,3 \%)$, as características definidoras citadas Quanto ao relato de apreensão foi à tensão aumentada em 43,3\% e nervosismo em $38,3 \%$, o principal fator relacionado em $74 \%$ foi separação do sistema de apoio em situação potencialmente estressante.

\section{DISCUSSÃO}

De acordo com o Boletim Epidemiológico, a faixa etária no sexo masculino (diagnosticados entre os anos de 1980 e 2006) mais acometida é a de 25 a 49 anos, na Qual estão 79\% dos casos da doença e no sexo feminino, de 20 a 49 anos, representando $28,8 \%$ do total de casos de aids do país segundo a faixa etária. Neste estudo, podemos observar Que a maioria dos pacientes era do sexo masculino (65\%), com idade média de 36 anos (26-46); a idade média do sexo feminino foi de 35 anos (23-64). No Brasil, verifica-se um adoecimento maior dos casos notificados de aids, na faixa etária de 20 a 49 anos $(83,4 \%)$, independente do sexo ${ }^{(2)}$.

A baixa escolaridade também ajuda na disseminação do vírus. A variável escolaridade tem sido utilizada como marcador da situação sócio-econômica, e o aumento na proporção de casos de aids naqueles indivíduos com menor escolaridade tem sido denominado pauperização do agravo ${ }^{(9)}$. Neste estudo, 38,3\% dos pacientes completaram o segundo grau ( 11 anos de estudo), seguido de primeiro grau incompleto (entre um a sete anos de estudo) 30\%; nossos achados foram semelhantes aos encontrados em outro estudo ${ }^{(10)}$, onde a baixa escolaridade relacionada ao primeiro grau incompleto predominou em $50 \%$ dos casos.

Atualmente no Brasil, a principal via de transmissão da doença é a relação heterossexual desprotegida, respondendo por $86,8 \%$ dos casos notificados em mulheres e por $25,7 \%$ dos casos em homens $^{(2)}$. Este estudo mostrou Que houve predominância de solteiros do sexo masculino com relação heterossexual $(64,1 \%)$.

Com relação ao estilo de vida, no Que diz respeito ao fator de risco, uso de drogas injetáveis (UDI), a maioria dos pacientes deste estudo relata nenhuma participação e/ou consumo $(58,3 \%)$. Entre os UDI, podemos observar Que 20,5\% eram homens e $14,3 \%$ mulheres e estes achados são similares aos dados do Boletim Epidemiológico, onde a segunda via de transmissão mais eficaz tem sido o compartilhamento de seringas entre os usuários de drogas injetáveis, Que responde por 22,8\% dos casos registrados no grupo masculino e por $5,7 \%$ entre as mulheres ${ }^{(2)}$.

As infecções oportunistas passam a surgir ou recidivar, tais como, tuberculose, pneumonia por Pneumocystis jiroveci, toxoplasmose cerebral, candidíase e meningite por criptococos, dentre outras. Em um estudo realizado em pacientes portadores do HIV/aids, atendidos em uma unidade ambulatorial no período 1993 - 200 I , a maior freqüência foi de tuberculose com $26 \%$, seguida da pneumocistose com $20,5 \%$ e toxoplasmose com $20,4 \%(10)$.

Os pacientes portadores de aids apresentam reações frente à doença e seu prognóstico. São problemas de saúde, Que geram respostas humanas físicas e emocionais, para os Quais os diagnósticos de enfermagem podem ter forte representação e contribuição tanto na prevenção como em sua recuperação.

Apesar das enfermeiras serem treinadas para as ações educativas, as mesmas não conseguem dar a mesma prioridade para os problemas emocionais do paciente como dão aos problemas físicos ${ }^{(11)}$, mas como pode ser observado em nosso estudo, a freqüência dos diagnósticos na esfera psicossocial ultrapassaram $70 \%$.

O enunciado completo de um diagnóstico contém o título (nome, rótulo) do mesmo, um conjunto de características definidoras e um ou mais fatores relacionados. Para melhor compreensão sobre as características definidoras elas são o conjunto de sinais e sintomas (indicadores) Que sustentam a afirmação de Que a resposta expressa no título está presente. Quanto aos fatores relacionados incluemse fatores do ambiente, da pessoa ou da integração de ambos Que favorecem a ocorrência da "resposta" expressa pelo título diagnóstico ${ }^{(18)}$.

Os elementos do enunciado de diagnósticos de risco (potenciais) sofrem algumas modificações se comparados a diagnósticos atuais. Nos diagnósticos potenciais os sinais ou sintomas ainda não se manifestaram; os dados ainda estão incompletos. No entanto, estes fatores colocam o paciente em risco, ou fazem a enfermeira suspeitar de Que um diagnóstico é possível.

Na AIDS plenamente manifesta, são freqüentes as septicemias bacterianas (infecção hospitalar) e disseminação das infecções oportunistas, Que conduzem o paciente a um estado toxêmico, com alterações hemodinâmicas e funcionais multissistêmicas graves $^{(12)}$. Percebemos, no presente estudo, Que são vários os fatores Que contribuíram para Que o diagnóstico risco de infecção estivesse presente em 100\% dos casos, sendo Que os procedimentos invasivos desde punção de acessos venosos periféricos e centrais, sondagem vesical e a própria imunidade, como imunossupressão causada por medicamentos, foram os fatores Que ocorreram em maior freeüência. Estudos relacionados a pacientes portadores do vírus da imunodeficiência humana também identificaram este diagnóstico de enfermagem, confirmando Que a defesa secundária inadequada está presente em $100 \%^{(13,14)}$.

O diagnóstico de enfermagem proteção ineficaz descreve a diminuição na capacidade de proteger-se contra ameaças internas e externas, como doenças ou lesões ${ }^{(7)}$. Estudo realizado em pacientes hospitalizados com aids, o diagnóstico proteção alterada esteve presente em $100 \%$ dos casos e estava relacionado aos distúrbios imunológicos e alterações hematológicas ${ }^{(15)}$. Estes dados são similares aos do presente estudo, onde observamos Que este diagnóstico esteve presente em 100\% dos casos, sendo Que estavam relacionados aos fatores sanguíneos, hemograma, leucograma alterados, seguidos de distúrbios da imunidade, ambos com 100\% de freqüência e nutrição inadęuada com $60 \%$.

O diagnóstico de enfermagem padrão de sono perturbado é definido por distúrbio com tempo limitado na Quantidade ou Qualidade do sono (suspensão natural, periódica da consciência) ${ }^{(7)}$. Pode ser evidenciado por diversos fatores: efeitos colaterais dos medicamentos, depressão, medo, internação hospitalar, vergonha, família e amigos - interação prejudicada, futuro, entre outros Que interferem no sono e repouso ${ }^{(16)}$.

Nossos resultados são semelhantes aos encontrados em um estudo com pacientes internados com afecções infecciosas e 
parasitárias, em Que este diagnóstico também esteve presente em $100 \%$ dos $\operatorname{casos}^{(15)}$. Distúrbios do sono por um período prolongado acarretam alterações do curso do pensamento, da concentração e da memória, irritabilidade e redução do tempo de resposta aos estímulos. Alterações do sono são sinais característicos de depressão, bem como, interações medicamentosas, sendo necessária a investigação para a resolução deste problema ${ }^{(1)}$.

O diagnóstico de enfermagem intolerância à atividade é evidenciado pela energia fisiológica ou psicológica insuficiente para suportar ou completar as atividades diárias reQueridas ou desejadas ${ }^{(7)}$. Alterações psicomotoras, sintomas precoces de demência, alterações no padrão respiratório e sistema nervoso central (neuropatias periféricas, parestesias), dentre outros, resultam em condições insatisfatórias restringindo o paciente, impedindo a deambulação e as atividades usuais $^{(12,17)}$. Em nosso estudo o mesmo esteve presente em 93,3\% dos casos e nota-se Que o principal fator relacionado foi deseeuilíbrio de oferta e demanda de oxigênio em 73,2\%.

O diagnóstico de enfermagem déficit no autocuidado para alimentação foi identificado em 91 ,6\% dos pacientes deste estudo. As alterações cognitivo-motoras ligadas ao HIV e outras anormalidades comportamentais, possivelmente ocorrem com maior freqüência, em indivíduos com infecção por HIV, como demonstrado neste estudo, onde o prejuízo perceptivo ou cognitivo teve uma fręüência de 50,9\% e prejuízo neuromuscular em 27,3\%. Embora sintomas cognitivos geralmente precedam o envolvimento motor, sinais e sintomas motores são freqüentes durante a evolução do processo. Há perda progressiva do controle manual e do equilíbrio, e acidentes são freQüentes ${ }^{(17)}$.

O diagnóstico de enfermagem ansiedade é caracterizado por um vago e incômodo sentimento de desconforto ou temor, acompanhado por uma resposta autonômica (a fonte é freqüentemente não específica ou desconhecida para o indivíduo); um sentimento de apreensão causado pela antecipação de perigo. É um sinal de alerta Que chama a atenção para um perigo iminente e permite ao indivíduo tomar medidas para lidar com a ameaça ${ }^{(7)}$.

A ansiedade é a manifestação psicológica mais comum em todas as fases da infecção pelo HIV. Pode apresentar, como sinais principais a in̨uietação, irritabilidade, pressão precordial, insônia e somatizações. A intensidade pode variar de acordo com o grau de informação do paciente e o acesso, maior ou menor, aos recursos terapêuticos. Há uma incidência maior de ansiedade nas pessoas vivendo com HIV/AIDS em relação à população geral. Modificações nos esquemas terapêuticos e variações dos resultados dos exames laboratoriais de controle podem se transformar em fatores ansiogênicos importantes ${ }^{(1)}$.

Neste estudo, o diagnóstico ansiedade esteve presente em $90 \%$ dos casos e observamos Que os principais fatores foram a ameaça pelo estado de saúde em 33,3\% e pela ameaça de morte em 66,6\%.

O diagnóstico de enfermagem conhecimento deficiente é definido por ausência ou deficiência de informação cognitiva relacionada a um tópico específico ${ }^{(7)}$.

A disfunção neurológica resulta dos efeitos diretos do HIV sobre o tecido do sistema nervoso e pode levar ao declínio progressivo nas funções cognitiva, comportamental e motoras ${ }^{(16)}$. Neste estudo, o referido diagnóstico esteve presente em $88,3 \%$ dos casos e o fator relacionado, falta de familiaridade com os recursos de informação, esteve presente em $56,6 \%$ e limitação cognitiva em 50,9\%. Estudo realizado por França ${ }^{(14)}$, mostra que a baixa ou nenhuma escolaridade e déficit de conhecimento sobre a patologia são frecüentes (80\%) e evidência e/ou dificuldade de acesso às informações igualitárias sobre saúde (recursos de informação) em 100\%.

As manifestações cutâneas estão associadas à infecção por HIV, às infecções oportunistas e malignidades acompanhantes, também ao estado nutricional, alterações psicomotoras e terapia medicamentosa, dentre outros. Independente da origem, os pacientes vivenciam desconforto e estão em risco aumentado de infecção adicional a partir da ruptura da integridade cutânea ${ }^{(16)}$.

Em estudo relacionado a pacientes com aids ${ }^{(14)}, \mathrm{o}$ diagnóstico de enfermagem risco de integridade da pele prejudicada esteve presente em $100 \%$ dos casos e estavam relacionados à alteração no estado nutricional, no turgor e elasticidade da pele, prurido, permanência prolongada no leito e fator imunológico. Em nosso estudo este diagnóstico esteve presente em 100\% dos casos, reforçando Que tanto os fatores externos como contenção e hipertermia atuam como preditores de risco para este diagnóstico como também os fatores internos, envolvendo imunidade, estado nutricional e uso de medicamentos, entre outros.

O diagnóstico de enfermagem padrão respiratório ineficaz é definido pela inspiração e/ou expiração Que não proporciona ventilação adeQuada $^{(7)} \mathrm{e}$ foi identificado em $85 \%$ dos pacientes e como principal fator relacionado podemos citar a síndrome da hipoventilação em $60,8 \%$. A respiração comprometida é uma importante complicação Que aumenta o desconforto e a ansiedade do paciente, podendo levar a insuficiência respiratória e a insuficiência cardíaca ${ }^{(16)}$. Uma das principais complicações da aids está relacionada com a presença de afecções Que atingem o sistema respiratório, sendo Que estas são as principais causas de morbidade e mortalidade em pacientes com infecção pelo $\mathrm{HIV}^{(1,12)}$.

O diagnóstico de enfermagem medo é a resposta à ameaça percebida Que é conscientemente reconhecida como um perigo ${ }^{(7)}$. No paciente portador de HIV/aids, o medo pode estar exacerbado por demandas ambientais, sociais ou internas ${ }^{(1)}$. Os pacientes, apesar de receberem instruções, orientações e apoio desde a internação, Que geralmente é prolongada, eles verbalizam o medo relacionado à progressão da doença e morte e o fato de a hospitalização ser um ambiente novo e estressante, gerando insegurança e temor pelo Que vai acontecer.

O diagnóstico de enfermagem risco de lesão significa estar em risco de lesão como resultado de condições ambientais interagindo com recursos adaptativos e defensivos do indivíduo ${ }^{(7)}$. Em nosso estudo, esse diagnóstico teve uma freqüência de $81,6 \%$ e está relacionado com disfunção auto-imune em $100 \%$ dos casos, má nutrição em $73,5 \%$, em situações de transporte do paciente (posicionamento) em 38,8\% e fragilidade da pele em 32,6\%. As manifestações cutâneas estão associadas à infecção por HIV, às infecções oportunistas e malignidades acompanhantes e com o estado nutricional, dentre outros fatores e a dor e o desconforto estão presentes a partir da ruptura da integridade cutânea ${ }^{(16)}$.

\section{CONSIDERAÇÕES FINAIS}

Neste estudo, nossos resultados permitiram concluir Que os pacientes portadores de HIV/AIDS apresentaram vários diagnósticos de enfermagem, confirmando o Que a experiência 
prática já apontava. Foram identificados trinta e oito diagnósticos de enfermagem, sendo Que onze estiveram presentes com uma freqüência superior a $80 \%$. Os fatores de risco e relacionados, de acordo com a taxonomia II da NANDA, foram suficientes para identificar os diagnósticos de enfermagem.

Nesta pesquisa, o Que se propõe é uma contribuição nessa área, estimulando o profissional para uma visão de ensino, pesQuisa e assistência servindo como referência bibliográfica e científica, voltada para a reflexão acerca da problematização da aids e gerando a construção do pensamento crítico do enfermeiro.

Os diagnósticos de enfermagem aqui identificados podem ser referência para discussão e análise, favorecendo uma enfermagem muito mais científica a Qual estamos buscando, conduzindo ao um cuidado cada vez mais aperfeiçoado.

\section{REFERÊNCIAS}

I. Rachid M, Schecter M. Manual de HIV/AIDS. 6 ${ }^{\mathrm{a}}$ ed. Editora Rio de laneiro: Revineter; 2001.

2. Ministério da Saúde (BR). Fundação Nacional da Saúde. Saúde divulga novos números da aids no Brasil. Dados epidemiológicos, CN - DST/AIDS. Bol Epidemiol AIDS/DST 2006; 3(1).

3. Souza MF. Teorias de enfermagem - Importância para a profissão. Acta Paul Enferm 1988; I (3): 63-65.

4. Braga CG, Cruz DALM. A Taxonomia II proposta pela North American Nursing Diagnosis Association (NANDA). Rev Latinoam Enfermagem 2003; II (02): 240-4.

5. Carpenito LJ. Diagnósticos de enfermagem, aplicação à prática clínica. 6a ed. Porto Alegre: Artes Médicas; 1997.

6. Cruz DALM. Fenômenos de enfermagem: desatando nós conceituais. In: Garcia TR, Nóbrega NML, organizadoras. Sistemas de classificação da prática de enfermagem: um trabalho coletivo. João Pessoa: ABEn; 2000. p.28-36.

7. North American Nursing Diagnosis Association. Nursing Diagnoses: definitions and classification: 2005/2006. Philadelphia: NANDA; 2001.

8. Doenges ME, Moorhouse MF. Diagnósticos e Intervenções em Enfermagem. 5a ed. Porto Alegre: Artmed; 1999.

9. Ministério da Saúde (BR). Fundação Nacional da Saúde. Doenças Infecciosas e Parasitárias - Guia de bolso. Aspectos clínicos, vigilância epidemiológica e medidas de controle. Brasília: Ministério da Saúde; 1999.

10. Gabriel R. Estudo epidemiológico de pacientes ambulatoriais com HIV/AIDS [dissertação]. São Paulo: Universidade Federal de São Paulo; 2003.

11. Carmagnani MIS. Diagnósticos e intervenções de enfermagem em pacientes submetidos à laringectomia total e parcial [tese]. São Paulo: Departamento de Enfermagem, Universidade Federal de São Paulo; 1999.

12. Veronesi R, Foccacia R. Tratado de infectologia. $2^{\text {a }}$ ed. São
Paulo: Atheneu; 2004.

13. Vaz MIR. Assistência de enfermagem às gestantes portadoras do vírus da imunodeficiência humana: levantamento de dados e diagnósticos de enfermagem [dissertação]. São Paulo: Escola de Enfermagem, Universidade de São Paulo; 1998.

14. França UM. Diagnósticos de enfermagem e uma proposta de intervenções para pacientes com AIDS [dissertação]. Ioão Pessoa: Universidade Federal da Paraíba; 1998.

15. Silva MR, Lima MT, Barros ABL. Principais diagnósticos de enfermagem segundo a NANDA e padrões funcionais de saúde com afecções Infecciosas e parasitárias. In: Exposição de Trabalho Científicos de Enfermagem. 200 I. São Paulo (SP), Brasil. São Paulo: Universidade Federal de São Paulo/UNIFESP; 2001 . p. 54.

16. Smeltzer SC, Bare BG. Brunner \& Suddarth - Tratado de enfermagem médico-cirúrgica. $9^{\mathrm{a}}$ ed. Rio de Janeiro: Guanabara-Koogan; 2002.

17. Schecter M, Marangoni DV. Doenças infecciosas: conduta diagnóstica e terapêutica. $2^{\text {a }}$ ed. Rio e Janeiro: GuanabaraKoogan; 1998.

18. Cruz DALM. A inserção do diagnóstico de enfermagem no processo assistencial. In: Cianciarullo TI, Gualda DMR, Melleiro MM, Anabuki MH, organizadoras. Sistema de Assistência de Enfermagem: evolução e tendências. São Paulo: Ícone Editora; 2001. p. 63-84.

19. UNAIDS (The joint United Nations Programe on HIV/AIDS). Report on the global AIDS epidemic: executive summary, December 2006. In: UNAIDS. 2006 report on the global AIDS epidemic: december 2006. [cited on 2007 set 18]. Disponível em: http://www.unaids.org/en/HIV_data/epi2006/ default.asp

20. Barbosa MS, Mafei FH, Marin MJS. Diagnósticos e intervenções de enfermagem aos pacienes em terapia anticoagulante. Rev Bras Enferm 2004; 57(5): 601-4. 